\title{
Hypofractionated Whole Breast Radiotherapy with Simultaneous Integrated Boost Following Breast Conservative Surgery for Early Breast Cancer
}

\author{
Ahmed R. Eldesoky, Ahmed H. Elshahat, Mona M. Eskander, Mona M. Fouda
}

\begin{abstract}
Department of Clinical Oncology and Nuclear Medicine Department, Faculty of Medicine, Mansoura University Egypt

Correspondence to: Ahmed R. Eldesoky, Department of Clinical Oncology and Nuclear Medicine Department, Faculty of Medicine, Mansoura University Egypt

email:
\end{abstract}

dr.aramadan85@yahoo.com

Received: 22 March 2020

Accepted: 29 March 2020

\section{Abstract:}

Background: Hypofractionated whole breast radiotherapy (HFWBRT) showed comparable efficacy and safety to conventional fractionated radiotherapy. Dose and fractionation of the tumor bed boost to be integrated during HF-WBRT schedules is still to be determined. Aim: to investigate the clinical feasibility of HF-WBRT with simultaneous integrated boost (SIB), in early breast cancer patients who underwent breast conserving surgery (BCS). Methods: This single arm prospective study included 40 female patients with pathologically proven stage I-II breast cancer following BCS with high risk factors. Patients received 3D conformal radiotherapy (3DCRT) with field in field (FIF) technique. The whole breast received a dose of 40 gray (Gy) over 15 fractions for 3 weeks with an additional SIB dose of 8 Gy over 15 fractions to be give daily during WBRT. Radiation toxicities were graded using the common terminology criteria for adverse events (NCI-CTCAE) scale version 4.03. Cosmetic outcome was assessed using Harvard cosmetic score. Kaplan Meier method was used to estimate the 3-year disease free (DFS) and overall survival (OS). Results: The median age at presentation was 48 years (range 33-68). No reported grade 3 or 4 toxicities. Grade 1 and 2 radiation dermatitis affected $80 \%$ of the patients, breast pain was observed in $62.5 \%$ of the patients while $25 \%$ had radiation pneumonitis. Most of the patients (95\%) had excellent and good cosmetic outcomes. At 3 years the estimated DFS was $95 \%$ and OS was $97.5 \%$. Conclusion: WBRT-SIB using 3DCRT with FIF technique is clinically feasible for early stage breast cancer patients.

Key words: Breast conservative therapy, hypofractionated radiotherapy, simultaneous integrated boost, Breast Cancer. 


\section{Introduction}

Breast carcinoma is the 2nd most common malignancy worldwide and the commonest cancer among women (1). It represented 30\% of all new cancer diagnoses (2). Breast conserving therapy (BCT) is the preferred treatment option for local management of early breast cancer. With a 15-year followup, a meta-analysis of 17 randomized trials including more than 10,000 women found an ipsilateral breast recurrences risk reduction by a factor of at least 3-4 after adding radiotherapy to $\mathrm{BSC}(3)$. This leads to a reduction in breast cancer deaths of about $3 \%$ and $8 \%$ for in node-negative and nodepositive patients respectively. Moreover, further evidence confirmed tolerable treatment-related early and late side effects and excellent cosmetic results (4).

Conventional adjuvant breast irradiation is usually given in two phases: whole breast radiotherapy (WBRT) for 5 weeks plus a tumor bed boost dose for 1-1.5 weeks. Positive margins and young patient age are the most important independent risk factors for local recurrences (LR) after BCT (5).

The boost dose is recommended in all patients with tumor-free margins of all age groups. In a trial conducted by the European organization of research and treatment of cancer, a total of 5,319 stage I and stage II patients after local excision and axillary dissection received the standard 50 Gray (Gy) WBRT; they were then randomized to $16 \mathrm{~Gy}$ boost vs. no further radiotherapy. At 5 years the LR rates were $7.3 \%$ for breast only and $4.3 \%$ for breast and boost radiotherapy groups, respectively $(\mathrm{p}<0.001)$. Patients younger than 40 years had the absolute gains in local control (6). More recently, the young boost trial reported the cosmetic outcome at four years. Factors associated with significant worse cosmesis included adjuvant chemotherapy, poor cosmesis before radiation therapy, a photon boost, large tumor bed volume, high boost dose (7).

From the radiobiological point of view, slow growing tumors such as breast with low $\alpha / \beta$ ratios, benefit from hypofractionated radiotherapy (HF-RT) (8). Randomized clinical trials enrolled more than 7,000 patients with early breast cancer for the HFWBRT arms (9). In the START B trial, 2,215 women were randomized after BCS or mastectomy either 50 Gy in 25 fractions over 5 weeks or the accelerated HF-RT regimen of 40 Gy in 15 fractions over 3 weeks to the whole breast. At 5 years, LR rate was 3.3\% and $2.2 \%$, survival rate was $87.5 \%$ and $90.4 \%$, and cosmetic differences rate was $42.5 \%$ and $36.5 \%$, in the standard and $\mathrm{HF}$ arms respectively (10). A Canadian trial showed that LR after 10-year follow-up was $6.7 \%$ and $6.2 \%$ in standard and HF arms, respectively. Ten-year survival rates were 
$84.4 \%$ and $84.6 \%$ in control and HF-RT groups, respectively. Good and excellent cosmetic outcomes at 10 years were similar; $71.3 \%$ in the control group and $69.8 \%$ in the HF- group (11).

Nowadays, the best tumor bed boost dose and fractionation to be integrated during $\mathrm{HF}$ WBRT schedules is still to be determined. The integration of the boost within the WBRT showed a dosimetric advantage for both target volumes and organs at risk (12). Prospective or retrospective studies provided their clinical results on WBRT with SIB. The ARO-201001 is a multicenter trial that found that the SIB schedule in early breast cancer patients after BCS was dosimetrically feasible (13).

Two large phase III prospective randomized trials are currently investigating HF-WBRT with SIB. The radiotherapy oncology group 1005 trial compared standard WBRT of 50 Gy/25 fractions followed by a sequential boost of 12-14 Gy/6-7 fractions versus a HFWBRT of 40 Gy/15 fraction (2.67 Gy daily) with a SIB of 3.2 Gy (48 Gy/15 fraction). This trial has recently finished accrual, and results are awaited (14). In the IMPORT High trial, a standard arm of $40.5 \mathrm{~Gy} / 15$ fractions (2.7 Gy daily) with a sequential boost of 16 Gy/8 fractions was compared to two experimental arms which included 36 Gy /15 fractions to the whole breast and 40 Gy/15 fractions to the affected quadrant, the first arm received $48 \mathrm{~Gy} / 15$ fractions, and the second arm received $53 \mathrm{~Gy} / 15$ fractions to the tumor bed (15).

The aim of the current study was to investigate the clinical feasibility of HFWBRT with daily SIB in early breast cancer patients who underwent BCS.

\section{Patients and Methods:}

The study protocol was granted approval by the institutional medical research ethical committee at Mansoura University at July 2014. This is a prospective non-comparative study that was conducted at Clinical Oncology and Nuclear Medicine Department, Mansoura University Hospital. The study included 40 patients with early stage breast cancer who underwent breast conserving surgery, followed by HF-WBRT with daily SIB. The study was conducted during the period from August 2014 till December 2017 with a median follow up of 18 months (range, 13-48 months).

\section{Patients}

The study included 40 female patients with pathologically proven stage I-II breast cancer following BCS with at least one of the following risk factors: age 18-50 years, grade 2-3 histology, positive axillary nodes, lymphovascular invasion, close or positive resection margins, extensive intraductal component, or hormone negative breast cancer. The patient was excluded if she had pathologic stages III or IV breast cancer, non- 
epithelial breast malignancies such as sarcoma or lymphoma, bilateral breast cancer, Paget's disease of the nipple, male breast cancer, sever or active respiratory or cardiac diseases, previous breast irradiation or Eastern Collaborative Oncology Group (ECOG) Scale of Performance Status (PS) of $>2$ (16).

\section{Methods}

\section{Pre-Treatment assessment}

Full history and physical examination was conducted at the time of study recruitment. Routine laboratory evaluation was done for all patients. Informed consent was signed by each patient. Chest X-ray was requested to all patients as a baseline study. Patients who had node positive disease had a post contrastcomputed tomography of the chest, abdomen and pelvis. Echocardiography was done for all patients who received chemotherapy and/or Trastuzumab. Bone scan was done for patients who had node positive disease or localized bony pains.

\section{Post- Treatment assessment and follow up:}

Radiotherapy adverse events (AEs) were scored using the NCI Common Terminology Criteria for Adverse Events (CTCAE) version 4.03 (17). All patients were reviewed weekly during radiotherapy treatment with for symptoms and signs of AEs. A breast examination was conducted at $1,3,6,9,12,18,24$ months post radiotherapy. Cosmetic outcome was assessed clinically and graded as (Excellent, Good, Fair, poor) according to Harvard cosmetic scale (18). Follow up plain x-ray and/or non-contrast chest computed tomography (CT) was done to patients who had $\geq$ grade 2 pneumonitis and echocardiography was done for patients who had cardiac diseases and/or received Trastuzumab.

\section{Radiotherapy treatment procedures:}

Radiation therapy started at a median of 11 weeks (range; 6-22 weeks) post surgery or last chemotherapy cycle. Simulation was performed with the patient in the supine position. Patients were positioned with breast boards for immobilization. A planning CT scan with an image thickness of $0.3 \mathrm{~cm}$ in the treatment position was obtained and sent from the diagnostic CT machine to the treatment planning system (Precise; Elekta). Radioopaque markers were used to identify the lumpectomy scar and the outline of the palpable breast tissue circumferentially. External skin localizing marks including permanent tattoos were performed for radiation daily localization and set-up accuracy.

Each of the target volumes and normal structures including ipsilateral lung and heart were delineated on each slice from the 3D planning $\mathrm{CT}$ in which that structure exists. Delineation was performed following the European Society of Radiotherapy and Oncology (ESTRO) consensus guidelines (19). 
A three dimensional conformal radiotherapy (3DCRT) plan was generated. Field in field (FIF) technique was used for better optimization of the plan. The lumpectomy boost was given by photon beams using additional $\geq 2$ beams. A SIB plan was generated for each patient. For the breast, a dose of 40 Gy in 15 fractions of 2.67 Gy per day was prescribed. For the tumor bed boost an additional $0.6 \mathrm{~Gy} /$ fraction for a total dose of 48.0 Gy in 15 fractions of 3.2 Gy per day was prescribed. Megavoltage photon beams with energies 6 and 15 MV were used according to breast size.

\section{Statistical Analysis}

Descriptive statistics including frequency, percent, mean and standard deviation (SD) or median and range were used as appropriate to describe patients, tumor and treatment characters. Toxicity scores were described using frequency and percent. Disease free and (DFS) overall survival (OS) at three years were estimated using Kaplan-Meier survival method. Two sided p-value was used to demonstrate significance. A p-value of less than 0.05 was considered significant. Statistical analysis was conducted using Stata software version 12.1 (StataCorp LP, Texas, USA).

\section{Results}

\section{Patients and disease characteristics}

The median age at presentation among the studied group was 48 years (range 33-68).
Twenty patients $(50 \%)$ were premenopausal while, 15 patients (37.5\%) were postmenopausal. All patients had an ECOG performance status scale of $\leq 1$. Most of patients were morbid obese with 31 patients $(78 \%)$ had a body mass index (BMI) of $>30$ at diagnosis (Table 1).

All patients underwent primary surgery followed by adjuvant systemic treatment and radiotherapy. Most of the patients (28 patients, 70\%) had pathologically stage T2 tumors with a mean size of $3.1 \mathrm{~cm} \pm 1.25$. Twenty two patients $(55 \%)$ had pathologically positive axillary nodes (pN1). Collectively, about 9 patients $(22.5 \%)$ had a pathological stage I and 31 patients ( $77.5 \%$ ) had a stage II disease. Eighteen patients (45\%) had grade II tumors, while 15 patients had grade III tumors (37.5\%). Only 8 patients (20\%) had primary tumors with associated lymphovascular space invasion (LVSI), and 2 patients (5\%) had extra-nodal tumor extension. Associated intraductal carcinoma was detected in 13 patients $(32.5 \%)$.

Immunohistochemistry (IHC) was used to examine both hormone receptors (HR) and Her2-neu gene. Thirty patients (75\%) had HR positive tumors, while only 6 patients $(15 \%)$ had positive Her2-neu staining. Using a cutoff of $20 \%$, Ki67 was low $(\leq 20 \%)$ in 19 patients $(47.5 \%)$ and high $(>20 \%)$ in 21 patients $(52.5 \%)$. Collectively, most of patients had 
luminal like tumors, while only $25 \%$ had more aggressive tumors.

\section{Treatment Characteristics}

Most of patients (38 patients; 95\%) had a wide local excision (lumpectomy) of their tumors. Only 2 patients (5\%) had complete quadrant excision (quadrantectomy), one of them because of a multifocal disease. Only one patient had Sentinel lymph node (LN) biopsy followed by axillary $\mathrm{LN}$ dissection (ALND). So, all patients underwent ALND.

Adjuvant chemotherapy was omitted in only 2 patients $(5 \%)$ because of old age and associated co-morbidities, while 38 patients (95\%) received a median of 6 chemotherapy cycles (range 4-16). Only one patient didn't complete her chemotherapy cycles. Most of patients (30 patients; $75 \%$ ) received the sequential regimen of anthracycline based followed by single agent taxane, others (10 patients; 25\%) received anthracycline containing chemotherapy. Adjuvant trastuzumab was planned for 6 patients with positive Her2-neu expression. However, only 2 patients received trastuzumab.

Thirty patients (75\%) with hormone sensitive tumors received adjuvant endocrine therapy according to their menopausal status. Tamoxifen was prescribed for 14 patients (35\%), while aromatase inhibitors (AIs) were prescribed for 13 patients $(32.5 \%)$. Only two patients $(5 \%)$ received Tamoxifen followed by an AI as a switch strategy. Only one premenopausal woman $(2.5 \%)$ received a combination of tamoxifen and medical ovarian ablation by monthly Goserlin (Table 2).

\section{Radiation induced AEs}

No grade 3 or 4 AEs were reported as shown in (Table 3). Radiation dermatitis was the commonest reported AEs in 32 patients (80 $\%)$. Dermatitis was frequently seen at the infra-mammary fold, axilla and the area at the junction between breast and $\mathrm{LN}$ beams. Twenty one patients (53\%) had localized pruritus. Breast pain was reported in 25 patients $(63 \%)$. Most of patients developed breast pain after the 2nd week of radiotherapy which lasted for a median of 6 weeks (range 2-11 weeks) after radiotherapy end.

Pneumonitis occurred only in 10 patients (25\%) and confirmed with chest imaging in 3 patients. Only one patient received short course of steroids. Dysphagia was reported in 19 patients (48\%), all of them received nodal irradiation. Only one patient developed an attack of chest pain which was considered as typical chest pain by emergency physician, supported by ECG changes. However, the patient was stable and went home on antiplatelet treatment. 
Table 1: Patients and disease Characteristics

\begin{tabular}{|c|c|c|}
\hline Variable & Number (40 patients) & $\%$ \\
\hline Age at presentation & Median 48 Y (range; 33-68). & 55 \\
\hline$\leq 50$ years & 22 & 45 \\
\hline$>50$ years & 18 & \\
\hline \multicolumn{3}{|l|}{ Menopausal status } \\
\hline Pre & 20 & 50 \\
\hline Peri & 5 & 12.5 \\
\hline Post & 15 & 37.5 \\
\hline \multicolumn{3}{|c|}{$\begin{array}{l}\text { Family history of breast } \\
\text { or ovarian cancer }\end{array}$} \\
\hline Positive & 6 & 15 \\
\hline Negative & 34 & 85 \\
\hline \multicolumn{3}{|l|}{ ECOG PS } \\
\hline 0 & 17 & 42.5 \\
\hline 1 & 23 & 57.5 \\
\hline BMI at presentation & Median 38 kg/m2 (range; 26-52) & \\
\hline$\leq 30$ & 9 & 22.5 \\
\hline$>30$ & 31 & 77.5 \\
\hline Tumor size & Mean $\pm \mathrm{SD}=3.1 \mathrm{~cm} \pm 1.25$ & \\
\hline \multicolumn{3}{|l|}{ Pathological T stage } \\
\hline $\mathrm{T} 1$ & 8 & 20 \\
\hline $\mathrm{T} 2$ & 28 & 70 \\
\hline T3 & 4 & 10 \\
\hline \multicolumn{3}{|l|}{ Pathological LN stage } \\
\hline No & 18 & 45 \\
\hline $\mathrm{N} 1$ & 22 & 55 \\
\hline \multicolumn{3}{|l|}{ Overall pathological } \\
\hline stage & 9 & 22.5 \\
\hline I & 31 & 77.5 \\
\hline \multicolumn{3}{|l|}{ II } \\
\hline \multicolumn{3}{|l|}{ Histological type } \\
\hline Ductal & 37 & 92.5 \\
\hline Lobular & 1 & 2.5 \\
\hline Others & 2 & 5 \\
\hline \multicolumn{3}{|l|}{ Histological grade } \\
\hline I & 7 & 17.5 \\
\hline II & 18 & 45 \\
\hline III & 15 & 37.5 \\
\hline \multicolumn{3}{|l|}{$\operatorname{Margins}(\geq 1 \mathrm{~cm})$} \\
\hline Negative & 40 & 100 \\
\hline Close & 0 & 0 \\
\hline Postive & 0 & 0 \\
\hline \multicolumn{3}{|l|}{ Extranodal infiltration } \\
\hline Positive & 2 & 5 \\
\hline Negative & 38 & 95 \\
\hline \multicolumn{3}{|l|}{ LVSI } \\
\hline Positive & 8 & 20 \\
\hline Negative & 32 & 80 \\
\hline \multicolumn{3}{|l|}{ Associated DCis } \\
\hline Positive & 13 & 32.5 \\
\hline Negative & 27 & 67.5 \\
\hline \multicolumn{3}{|l|}{ Hormone receptor by } \\
\hline IHC & 30 & 75 \\
\hline Positive & 10 & 25 \\
\hline \multicolumn{3}{|l|}{ Negative } \\
\hline Her2neu gene buy If & & \\
\hline Positive & 6 & 15 \\
\hline Negative & 34 & 85 \\
\hline Ki67 (Cutoff 20\%) & Median $($ range $)=25(10-90)$. & \\
\hline Low $(\leq 20 \%)$ & 19 & 47.5 \\
\hline High $(>20 \%)$ & 21 & 52.5 \\
\hline Intrinsic subtype (IH & & \\
\hline Luminal A & 16 & 40 \\
\hline Luminal B (Her2-ve) & 10 & 25 \\
\hline Luminal B (Her2+ve) & 4 & 10 \\
\hline Her2 enriched & 2 & 5 \\
\hline Triple negative & 8 & 20 \\
\hline
\end{tabular}

Table 2: Treatment characteristics

\begin{tabular}{|c|c|c|}
\hline Variable & $\begin{array}{l}\text { Number (40 } \\
\text { patients) }\end{array}$ & $\%$ \\
\hline \multicolumn{3}{|l|}{ Type of surgery } \\
\hline Lumpectomy & 38 & 95 \\
\hline Quadrantectomy & 2 & 5 \\
\hline \multicolumn{3}{|l|}{ Oncoplastic Surgey } \\
\hline Yes & 4 & 10 \\
\hline No & 36 & 90 \\
\hline \multicolumn{3}{|l|}{ Axillary staging and } \\
\hline management & 1 & 2.5 \\
\hline SLNB & 40 & 100 \\
\hline \multicolumn{3}{|l|}{ ALND } \\
\hline \multicolumn{3}{|l|}{ Adjuvant chemotherapy } \\
\hline Yes & 38 & 95 \\
\hline No & 2 & 5 \\
\hline Adjuvant Trastuzumab & Eligible patients $=6$ & \\
\hline Yes & 2 & 5 \\
\hline No & 38 & 95 \\
\hline Adjuvant Endocrine & Eligible patients $=30$ & \\
\hline therapy & 30 & \\
\hline Yes & 10 & 75 \\
\hline No & & 25 \\
\hline Type of endocrine therapy & According to decision & \\
\hline Tamoxifen only & 14 & 35 \\
\hline AIs only & 13 & 32.5 \\
\hline Switch & 2 & 5 \\
\hline $\begin{array}{l}\text { Tamoxifen + Ovarian } \\
\text { suppression (Medical) }\end{array}$ & 1 & 2.5 \\
\hline
\end{tabular}

Table 3: Toxicity scores using CTCAE 4.03

\begin{tabular}{|c|c|c|c|}
\hline Toxicity & Grade 0 & Grade 1-2 & Grade 3-4 \\
\hline $\begin{array}{l}\text { Radiation } \\
\text { dermatitis }\end{array}$ & $8(20 \%)$ & $32(80 \%)$ & 0 \\
\hline Pruritis & $19(47.5 \%)$ & $21(52.5 \%)$ & 0 \\
\hline Breast Pain & $15(37.5 \%)$ & $25(62.5 \%)$ & 0 \\
\hline Cough & $29(72.5 \%)$ & $11(27.5 \%)$ & 0 \\
\hline Dyspnea & $36(90 \%)$ & $4(10 \%)$ & 0 \\
\hline Pneumonitis & $30(75 \%)$ & $10(25 \%)$ & 0 \\
\hline Dysphagia & $21(52.5 \%)$ & $19(47.5 \%)$ & 0 \\
\hline Cardiac & $39(97.5 \%)$ & $1(2.5 \%)$ & 0 \\
\hline
\end{tabular}

\section{Post treatment Cosmetic outcomes}

Most of the patients (95\%) had excellent or good post radiation cosmetic outcome by Harvard scale (Fig 1).

Twenty five patients $(62.5 \%)$ had excellent cosmetic outcome, while 13 patients (32.5\%) 
had good cosmetic outcome. Only 2 patients (5\%) had fair or poor outcome, one of them had a primary oncoplastic surgery.

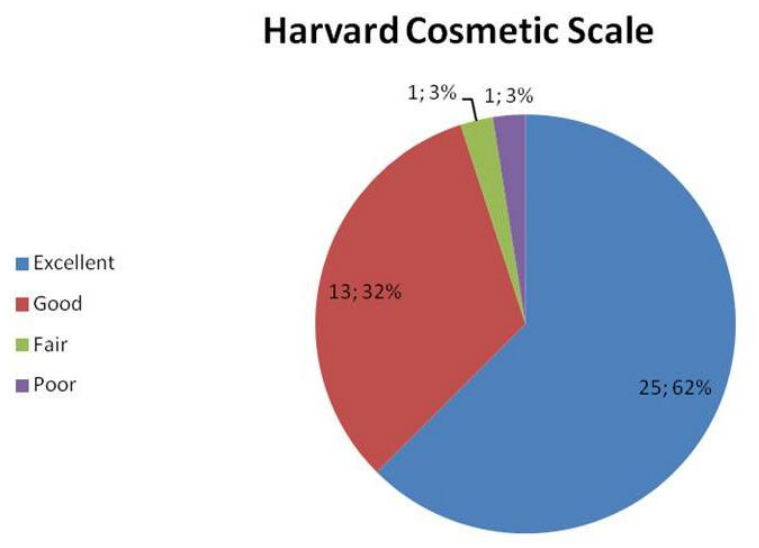

Figure 1. Pie chart for cosmetic outcome grading by Harvard grading scale.

\section{Local and distant failure analysis}

No patient developed local or regional relapse with local control rate of $100 \%$. Among the included patients two failure events were reported. One patient developed contra-lateral breast cancer at 25 months of her follow up. Another patient developed bone metastases at 48 months of her follow up.

\section{Survival outcomes}

One patient died because of cerebrovascular stroke at 24 months of her follow up. At 3years, local recurrence free survival, DFS and OS was $100 \%, 95 \%$ and $97.5 \%$ respectively (Fig 2\&3).

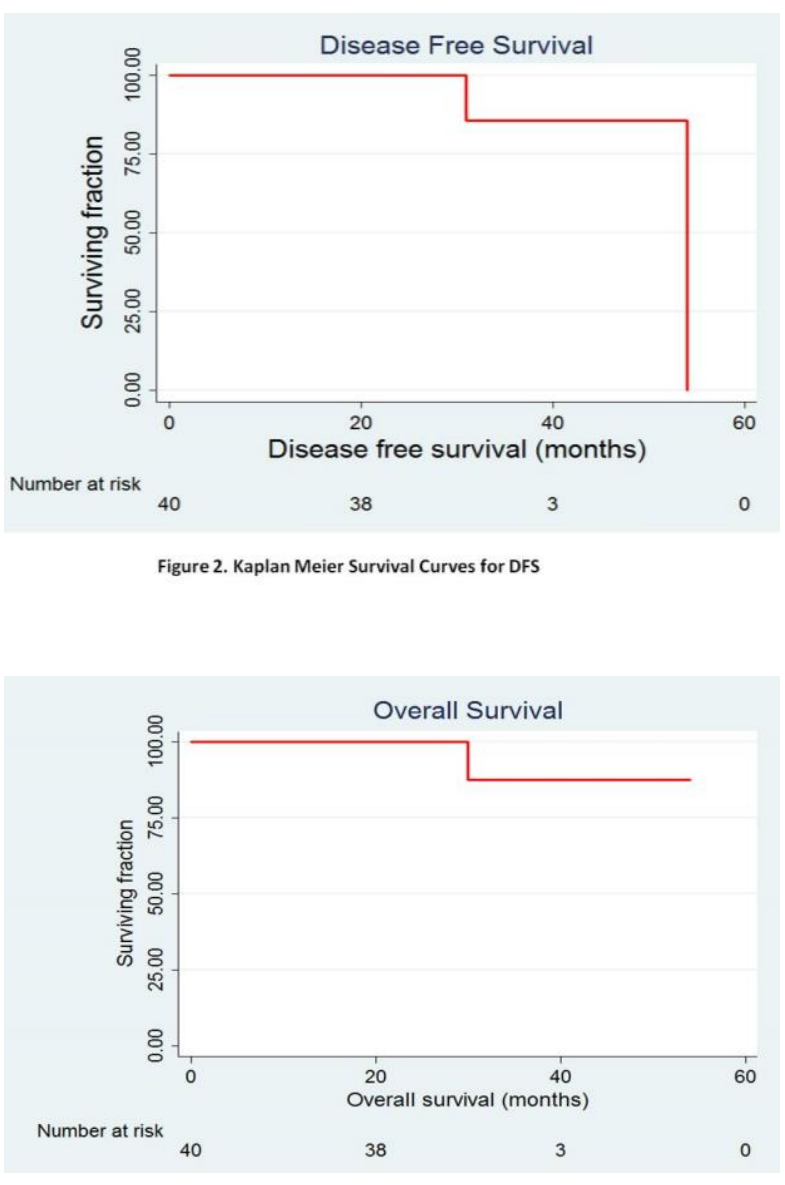

Figure 3: Kaplan Meier survival curves for OS

\section{Discussion}

Hypofractionation is a useful option for patients and healthcare providers. Potential advantages of $\mathrm{HF}$ are better patients convenience, faster patients turnover at busy radiotherapy departments and lower health related costs (20). The timing of combining HF-WBRT and tumor bed boost has not been determined yet. One of these strategies is to give a daily SIB with HF-WBRT in order to maintain the benefits of shortening the overall treatment time (12).

The current study included 40 patients who underwent BCS with high risk factors of local 
recurrence. WBRT-SIB plans were generated using 3D conformal FIF technique. Dermatitis was reported as the commonest AE. No reported grade 3 or 4 AEs. Grade $1 \& 2$ dermatitis was reported in 32 patients, 26 (65\%) had grade 1 and 6 (15\%) developed grade 2. Another protocol delivering $40.5 \mathrm{~Gy}$ to the breast and 45 Gy as a SIB in 15 fractions was investigated by Chadha et al. Early and late toxicity were low without any grade 3 or 4 skin toxicity after 2 years and grade 2 skin toxicity in 2 patients (4\%) (21).

A study included 75 patients with early-stage breast cancer. The breast received 45 Gy with a concurrent electron boost dose of $56 \mathrm{~Gy}$ over 4 weeks. They reported early skin toxicity as grade 0 in 9 patients $(12 \%)$, grade 1 in 49 patients (65\%), and grade 2 in 17 patients (23\%) (22). Formenti et al. included 91 women treated in prone position into a single arm prospective study of WBRT to 40.5 Gy/15 fractions over 3 weeks with a daily SIB of $45 \mathrm{~Gy} / 15$ fractions. After a median follow up of 12 months, 2 patients (2.1\%) had acute grade 3 and $67 \%$ of patients developed grade 1-2 dermatitis (23).

In the present study, 21 patients $(58 \%)$ had $\geq$ grade 1 pruritus. A Belgian randomized study treated patients in prone position to 40.05 Gy/15 fractions to the whole breast. A sequential boost of $10 \mathrm{~Gy} / 4$ fractions or 14.88 Gy/6 fractions was prescribed for close negative or positive surgical margins respectively in the control arm. In the experimental arm a SIB of 46.8 or 49.95 Gy was prescribed for negative and positive surgical margins, respectively. Fifty-one patients $(61 \%)$ in the SIB arm developed pruritus (24).

In the current study, 10 patients (25\%) developed $\geq$ grade 1 lung toxicity. In Van Parijs et al. report, 69 women were randomized between conventional radiotherapy 50 Gy/25 fractions, and sequential boost $16 \mathrm{~Gy} / 8$ fractions if $\mathrm{BCS}$ versus experimental HF tomotherapy 42 Gy/15 fractions and SIB of 0.6 Gy if BCS (cumulative dose $51 \mathrm{~Gy} / 15$ fractions). Change in forced expiratory volume in one second (FEV1) and diffusing capacity of the lung for carbon monoxide (DLco) were reported. Lung function tests showed a significant reduction in HF arm based on changes of DLco, $\mathrm{P}=$ 0.047 , but not on changes of FEV1. At 2 years, 5 patients $(22 \%)$ in the experimental arm had $\geq$ grade 1 lung toxicity (25).

Regarding cosmetic outcome (CO), 95\% and $5 \%$ of the current study participants had a good or excellent, and fair or poor cosmesis respectively. Franco et al. reported good/excellent CO in $91 \%$ and fair/poor in 9 $\%$ of patients (26). Cosmesis were good to excellent in all the patients included in a study by Mondal et al. using Harvard grading scale (27).

In the present study, a local control rate of $100 \%$ was reported. Two patients had 2 failure events. One of them developed distant 
bone metastases the other developed contralateral breast cancer. The estimated 3year DFS and OS was $95 \%$ and $97.5 \%$ respectively. Formenti et al. reported 1 recurrence among 91 breast cancer women who received SIB radiotherapy (23). Another study reported that 3-year loco-regional control, recurrence-free survival, and OS rates were 99.2, 95.5, and 97.1\%, respectively (28). Cante et al. used a dose of 45 Gy/20 fractions to the whole breast and a daily SIB dose 0.25 Gy to the tumor bed to a total dose of $50 \mathrm{~Gy}$. With a median follow up of 60 months, OS was $97.6 \%$; cancer-specific survival was 99.4\%; DFS was 96.6\%; and local control was $100 \%(\mathbf{2 9})$.

Limited resources are challenging to provide breast cancer radiotherapy in Africa including Egypt. There is often extreme burden leading to delayed treatment. Moreover, frequent interruption of radiotherapy treatments due to the costs of traveling for therapy can be significant (30, 31). Progressive introduction of shortened radiotherapy regimens like the one proposed in this study may effectively contribute to improved compliance and access to adjuvant breast cancer radiotherapy in areas with limited radiotherapy facilities.

The present study has some limitations which should be considered when interpreting the results. The non randomized single arm design, the small sample size and limited follow up period. However, our study achieved its aim which confirmed that HF-
WBRT with SIB is a feasible and safe treatment.

\section{Conclusion:}

The approach investigated in the current study allowed us to treat patients in three weeks period which was convenient to patients. All patients completed their treatment schedule with no interruption due to toxicity. This also reduced work load on the used equipments by decreasing number of treated patients per day.

\section{Conflict of interest}

The authors declare that they have no conflict of interest.

\section{References}

1. Bray F, Ferlay J, Soerjomataram I, Siegel Rl, Torre La, Jemal A. Global Cancer Statistics 2018: Globocan Estimates Of Incidence And Mortality Worldwide For 36 Cancers In 185 Countries. Ca Cancer J Clin 2018; 68: 394-424.

2. Siegel R1, Miller Kd, Jemal A. Cancer Statistics, 2017. Ca Cancer J Clin 2017; 67: 7-30.

3. Darby S, Mcgale P, Correa C, Taylor C, Arriagada R, Clarke M, Et Al. Effect Of Radiotherapy After Breast-Conserving Surgery On 10-Year Recurrence And 15-Year Breast Cancer Death: Meta-Analysis Of Individual Patient Data For 10,801 Women In 17 Randomised Trials. Lancet 2011; 378: 1707-1716.

4. Sedlmayer F, Sautter-Bihl Ml, Budach W, Dunst J, Fastner G, Feyer P, Et Al. Degro Practical Guidelines: Radiotherapy Of Breast Cancer I: Radiotherapy Following Breast Conserving Therapy For Invasive Breast Cancer. Strahlenther Onkol 2013; 189: 825-833. 
5. Houssami N, Macaskill P, Marinovich Ml, Morrow M. The Association Of Surgical Margins And Local Recurrence In Women With EarlyStage Invasive Breast Cancer Treated With BreastConserving Therapy: A Meta-Analysis. Ann Surg Oncol 2014; 21: 717-730.

6. Bartelink H, Maingon P, Poortmans P, Weltens C, Fourquet A, Jager J, Et Al. Whole-Breast Irradiation With Or Without A Boost For Patients Treated With Breast-Conserving Surgery For Early Breast Cancer: 20-Year Follow-Up Of A Randomised Phase 3 Trial. Lancet Oncol 2015; 16: 47-56.

7. Brouwers Pjam, Van We, Bartelink H, Fourquet A, Lemanski C, Van Lj, Et Al. Predictors For Poor Cosmetic Outcome In Patients With Early Stage Breast Cancer Treated With Breast Conserving Therapy: Results Of The Young Boost Trial. Radiother Oncol 2018; 128: 434-441.

8. Van Leeuwen $\mathrm{Cm}$, Oei $\mathrm{Al}$, Crezee J, Bel A, Franken Nap, Stalpers Lja, Et Al. The Alfa And Beta Of Tumours: A Review Of Parameters Of The Linear-Quadratic Model, Derived From Clinical Radiotherapy Studies. Radiat Oncol 2018; 13: 96.

9. Theberge V, Whelan T, Shaitelman Sf, Vicini Fa. Altered Fractionation: Rationale And Justification For Whole And Partial Breast Hypofractionated Radiotherapy. Semin Radiat Oncol 2011; 21: 5565 .

10. Bentzen Sm, Agrawal Rk, Aird Eg, Barrett Jm, Barrett-Lee $\mathrm{Pj}$, Bentzen $\mathrm{Sm}$, Et Al. The Uk Standardisation Of Breast Radiotherapy (Start) Trial B Of Radiotherapy Hypofractionation For Treatment Of Early Breast Cancer: A Randomised Trial. Lancet 2008; 371: 1098-1107.

11. Whelan Tj, Pignol Jp, Levine Mn, Julian Ja, Mackenzie R, Parpia S, Et Al. Long-Term Results Of Hypofractionated Radiation Therapy For Breast Cancer. N Engl J Med 2010; 362: 513-520.

12. Franco P, Cante D, Sciacero P, Girelli G, La Porta Mr, Ricardi U. Tumor Bed Boost Integration During Whole Breast Radiotherapy: A Review Of The Current Evidence. Breast Care (Basel) 2015; 10: 44-49.

13. Dellas K, Vonthein R, Zimmer J, Dinges S, Boicev Ad, Andreas P, Et Al. Hypofractionation With Simultaneous Integrated Boost For Early Breast Cancer: Results Of The German Multicenter Phase Ii Trial (Aro-2010-01). Strahlenther Onkol 2014; 190: 646-653.
14. Rtog. Rtog 1005: A Phase Iii Trial Of Accelerated Whole Breast Irradiation With Hypofractionation Plus Concurrent Boost Versus Standard Whole Breast Irradiation Plus Sequential Boost For EarlyStage Breast Cancer. Www Rtog Org 2014 [Cited 2020 Feb 4];

15. Tsang Y, Ciurlionis L, Kirby Am, Locke I, Venables K, Yarnold Jr, Et Al. Clinical Impact Of Import High Trial (Cruk/06/003) On Breast Radiotherapy Practices In The United Kingdom. Br J Radiol 2015; 88: 20150453.

16. Oken Mm, Creech Rh, Tormey Dc, Horton J, Davis Te, Mcfadden Et, Et Al. Toxicity And Response Criteria Of The Eastern Cooperative Oncology Group. Am J Clin Oncol 1982; 5: 649655.

17. Ctcae Version 4.03. Ctcae Version 4.03. Https://Evs Nci Nih Gov/Ftp1/Ctcae/Ctcae_4 03/Ctcae_4 03_2010-06-14_Quickreference_8 5x11 Pdf 2010 [Cited 2015 Nov 2];

18. Rose Ma, Olivotto I, Cady B, Koufman C, Osteen R, Silver B, Et Al. Conservative Surgery And Radiation Therapy For Early Breast Cancer. LongTerm Cosmetic Results. Arch Surg 1989; 124: 153-157.

19. Offersen Bv, Boersma Lj, Kirkove C, Hol S, Aznar Mc, Biete Sa, Et Al. Estro Consensus Guideline On Target Volume Delineation For Elective Radiation Therapy Of Early Stage Breast Cancer. Radiother Oncol 2015; 114: 3-10.

20. Lievens Y. Hypofractionated Breast Radiotherapy: Financial And Economic Consequences. Breast 2010; 19: 192-197.

21. Chadha M, Woode R, Sillanpaa J, Lucido D, Boolbol Sk, Kirstein L, Et Al. Early-Stage Breast Cancer Treated With 3-Week Accelerated WholeBreast Radiation Therapy And Concomitant Boost. Int J Radiat Oncol Biol Phys 2013; 86: 40-44.

22. Freedman Gm, White Jr, Arthur Dw, Allen L, X, Vicini Fa. Accelerated Fractionation With A Concurrent Boost For Early Stage Breast Cancer. Radiother Oncol 2013; 106: 15-20.

23. Formenti Sc, Gidea-Addeo D, Goldberg Jd, Roses Df, Guth A, Rosenstein Bs, Et Al. Phase I-Ii Trial Of Prone Accelerated Intensity Modulated Radiation Therapy To The Breast To Optimally Spare Normal Tissue. J Clin Oncol 2007; 25: 2236-2242.

24. Paelinck L, Gulyban A, Lakosi F, Vercauteren T, De Gw, Speleers B, Et Al. Does An Integrated 
Boost Increase Acute Toxicity In Prone Hypofractionated Breast Irradiation? A Randomized Controlled Trial. Radiother Oncol 2017; 122: 30-36.

25. Van Parijs H, Miedema G, Vinh-Hung V, Verbanck S, Adriaenssens N, Kerkhove D, Et Al. Short Course Radiotherapy With Simultaneous Integrated Boost For Stage I-Ii Breast Cancer, Early Toxicities Of A Randomized Clinical Trial. Radiat Oncol 2012; 7: 80.

26. Franco P, Zeverino M, Migliaccio F, Cante D, Sciacero P, Casanova B, V, Et Al. IntensityModulated And Hypofractionated Simultaneous Integrated Boost Adjuvant Breast Radiation Employing Statics Ports Of Tomotherapy (Tomodirect): A Prospective Phase Ii Trial. J Cancer Res Clin Oncol 2014; 140: 167-177.

27. Mondal D, Julka Pk, Sharma Dn, Jana M, Laviraj Ma, Deo Sv, Et Al. Accelerated Hypofractionated Adjuvant Whole Breast Radiation With Simultaneous Integrated Boost Using Volumetric Modulated Arc Therapy For Early Breast Cancer: A Phase I/Ii Dosimetric And Clinical Feasibility Study From A Tertiary Cancer Care Centre Of India. J Egypt Natl Canc Inst 2017; 29: 39-45.
28. Bantema-Joppe Ej, Vredeveld Ej, De Bock Gh, Busz Dm, Woltman-Van Im, Dolsma Wv, Et Al. Five Year Outcomes Of Hypofractionated Simultaneous Integrated Boost Irradiation In Breast Conserving Therapy; Patterns Of Recurrence. Radiother Oncol 2013; 108: 269-272.

29. Cante D, Franco P, Sciacero P, Girelli G, Marra Am, Pasquino M, Et Al. Five-Year Results Of A Prospective Case Series Of Accelerated Hypofractionated Whole Breast Radiation With Concomitant Boost To The Surgical Bed After Conserving Surgery For Early Breast Cancer. Med Oncol 2013; 30: 518.

30. Abdel-Wahab M, Bourque Jm, Pynda Y, Izewska J, Van Der Merwe D, Zubizarreta E, Et Al. Status Of Radiotherapy Resources In Africa: An International Atomic Energy Agency Analysis. Lancet Oncol 2013; 14: E168-E175.

31. Vanderpuye V, Grover S, Hammad N, Poojaprabhakar, Simonds H, Olopade F, Et Al. An Update On The Management Of Breast Cancer In Africa. Infect Agent Cancer 2017; 12: 13.

To cite this article: Ahmed R. Eldesoky, Ahmed H. Elshahat, Mona M. Eskander, Mona M. Fouda. Hypofractionated Whole Breast Radiotherapy with Simultaneous Integrated Boost Following Breast Conservative Surgery for Early Breast Cancer. BMFJ 2020; 37(1):259270. DOI:10.21608/bmfj.2020.26257.1233 